\title{
A Grounded MoM-based Spatial Green's Function Technique for the Analysis of Multilayered Circuits in Rectangular Shielded Enclosures
}

\author{
J. S. Gomez-Diaz ${ }^{1}$, Student Member, IEEE, M. Garcia-Vigueras ${ }^{1}$ Student Member, IEEE, \\ A. Alvarez-Melcon ${ }^{1}$, Senior Member, IEEE
}

\begin{abstract}
A continuous counterpart of the Spatial Images technique is proposed for the computation of the multilayered boxed Green's functions and, for the first time, of their derivatives. The method employs a set of auxiliary linear distribution of sources to effectively impose the potential boundary conditions along the whole cavity contour. The imposition of these boundary conditions leads for the first time to a set of integral equations (IEs), on the unknown distributions of the auxiliary sources, which are solved by applying a method of moments approach. A convergence/efficiency study, related to the test and basis functions choice, is then presented and discussed. The technique is combined with the use of dynamic ground planes generating mirror basis functions which completely remove any singular instability. Finally, the computed Green's functions are included into a mixed potential IE formulation for the accurate and very fast analysis of practical multilayered shielded circuits. The proposed technique does not suffer from any convergence issue and it is extremely competitive in terms of accuracy and efficiency as compared to other methods known to the authors.
\end{abstract}

Index Terms-Green's functions, Method of Moments (MoM), Integral Equations (IEs), Boxed circuits, Microwave filters.

\section{INTRODUCTION}

$\mathbf{T}$ HE ACCURATE and fast analysis of boxed multilayered circuits is required in the current design of many monolithic microwave integrated circuits (MMICs). The use of closed cavities provides physical support to microwave devices, immunity against electromagnetic interferences and avoids unwanted radiation. However, shielding enclosures also introduces additional effects that must rigorously be taken into account [1].

One efficient possibility for the analysis of these boxed devices is to employ the integral equation (IE) technique [2], solved by the method of moments (MoM) [3]. The IE method can be formulated either in the spectral [4], [5], [6], [7], [8] or in the spatial domain [9], [10], [11]. The spectral domain is usually very efficient, but it presents important convergence problems when the dimensions of the cells employed to discretize the printed circuits are very small as compared to the enclosure. Although some very efficient acceleration techniques have been proposed for 1D printed structures [8], the analysis of 2D metalizations still represents and interesting challenge. On the other hand, the spatial domain usually expresses the boxed multilayered Green's functions in terms of infinite sums of spatial images, which are very slowly

\footnotetext{
${ }^{1}$ Technical University of Cartagena, Campus Muralla del Mar s/n, Cartagena, E-30202, Spain, E-mail: jsebastian_gomez@ono.com
}

convergent. Another type of spatial-domain approach uses a discrete set of auxiliary point sources to impose boundary conditions on the potentials along discrete points on the cavity contour [12], [13]. This technique is able to efficiently analyze microwave shielded circuits but its stability strongly depends on the adequate selection of the auxiliary sources positions, as discussed in [13]. Besides, its discrete nature limits the accuracy of the computed Green's functions.

This paper presents the continuous counterpart of the discrete spatial-domain technique presented in [12], [13], particularized to the computation of the rectangular boxed multilayered Green's functions and, for the first time, to their associated derivatives. The continuous nature of the technique increases the accuracy that can be obtained in the Green's functions computation, with respect to other implementations based on discrete sources [12], [13]. Specifically, arbitrarily small errors in the Green's functions computation can be achieved. A refinement in the technique, which exploits the decoupling of the $x$ and $y$-dyadic components of the Green's functions in rectangular boxes, contributes to further improve the method efficiency.

Instead of discrete auxiliary point sources as in [13], the proposed continuous method uses a set of auxiliary linear distribution of sources to impose potentials boundary conditions along the whole cavity contour. After applying boundary conditions, a set of IEs, on the unknown values of the auxiliary sources, is obtained. The IE problem is then solved by using the MoM [3]. A rigorous study about the impact of the test and basis functions choice on the Green's functions convergence is then presented and discussed, showing a trade-off between accuracy (using roof-top basis/test functions) and speed (using point-matching basis/test functions). The concept of dynamic ground planes [14] is applied to the basis functions, generating mirror basis functions which completely solves any singular situation. Finally, the proposed theory is included into a mixed potential IE approach (MPIE) [2] and it is applied to the analysis of practical shielded microwave circuits, with planar metal patches printed at the dielectric interfaces. Two shielded microstrip filters are then analyzed and compared against other approaches and with measurements, showing excellent agreement. A careful comparative study demonstrates that the proposed method is extremely competitive as compared with other IE techniques known to the authors, avoiding any convergence problems. 


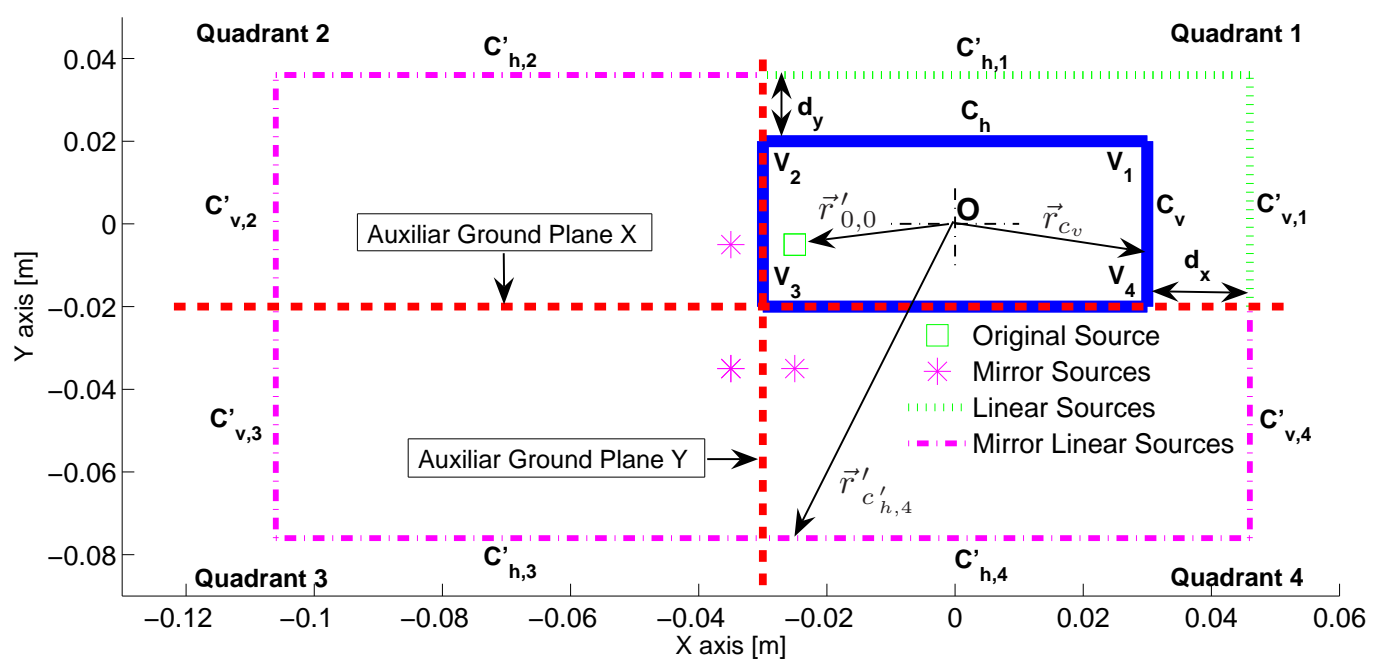

Fig. 1: An auxiliary linear distribution of sources $\left(C_{h, 1}^{\prime}\right.$ and $\left.C_{v, 1}^{\prime}\right)$ is combined with two auxiliary ground planes to analyze a multilayered rectangular enclosure. Mirror linear sources, with respect to the ground planes, appear from the original set of linear sources. Potential boundary conditions are then numerically imposed along the non-covered cavity walls, and are perfectly imposed along the covered walls. The dimensions of the cavity are $60 x 40 \mathrm{~mm}$, and it is composed of 2 layers: a dielectric layer $\left(\epsilon_{r}=2.2\right.$ of thickness $\left.3.17 \mathrm{~mm}\right)$, and an air layer $(3.0 \mathrm{~mm}$ height). The source is placed at the position $(-25,-5,3.14) \mathrm{mm} . O$ is the coordinates origin and cavity center.

\section{Grounded Mom-Based Spatial Technique}

This section first carefully derives the grounded MoMbased formulation. Then, the method is extended to obtain the $n$-order boxed Green's functions derivatives. Later, the location and definition of test and basis functions are carefully analyzed.

\section{A. Theoretical Overview}

Let us consider a multilayered rectangular cavity, which is excited by a point source. The first task is to obtain the Green's functions related to an infinite multilayered medium. This is easily accomplished using the Sommerfeld transformation [15] applied to the corresponding spectral domain Green's functions $(\tilde{G})[16]$.

The next step is to introduce a set of auxiliary distributions of linear sources. These sources are located surrounding the cavity under analysis (following the contour $C^{\prime}$ shown in Fig. 1). Here, the term linear is employed to emphasize that a continuous distribution of sources (such as 1D wires) is used, instead of discrete punctual sources as was the case of [12],[13]. The auxiliary linear sources are applied to compute both, the electric scalar and the magnetic vector potentials. In each case, the physical nature of the auxiliary linear sources corresponds to the potential under analysis (charge for the electric scalar potential and dipole currents for the magnetic vector potential). If the height of the cavity is electrically large, a set of discrete rings of auxiliary linear sources may be used [13], [17]. The unknown auxiliary distribution of sources are then computed to impose, in conjunction with the original point source, the boundary conditions on the lateral walls. Finally, the Green's functions inside the multilayered rectangular enclosure are recovered with the standard convolution integrals on the relevant sources of the problem

$$
\begin{aligned}
G_{V_{\text {rect }}}\left(\vec{r}, \vec{r}_{0,0}^{\prime}\right)= & S_{0}\left[\tilde{G}_{V}(\rho)\right]+ \\
& \int_{C^{\prime}} S_{0}\left[\tilde{G}_{V}(\rho)\right] Q\left(\vec{r}^{\prime}\right) \partial \vec{r}^{\prime}, \\
G_{A_{\text {rect }}}^{x x}\left(\vec{r}, \vec{r}_{0,0}^{\prime}\right)= & \left.S_{0}\left[\tilde{G}_{A}\right]\right|_{\vec{r}_{0,0}^{\prime}} ^{\vec{r}}+ \\
& \left.\int_{C^{\prime}} S_{0}\left[\tilde{G}_{A}\right]\right|_{\vec{r}^{\prime}} ^{\vec{r}} D\left(\vec{r}^{\prime}\right) \partial \vec{r}^{\prime},
\end{aligned}
$$

where $Q\left(\vec{r}^{\prime}\right)$ and $D\left(\vec{r}^{\prime}\right)$ are the auxiliary set of linear charges and dipoles, respectively. Also, $\left(S_{0}\right)$ denotes the zero-th order Sommerfeld transformation, applied to the spectral domain Green's functions for a specific source $\left(\vec{r}^{\prime}\right)$ and observer point $(\vec{r})$ locations. Note that a similar expression can easily be derived for a $y$-oriented source dipole.

The auxiliary linear sources are combined with the use of dynamic ground planes, a concept introduced in [14]. This allows to completely remove any singular instability produced by the point source when it is placed close to a cavity wall. Following this approach, ground planes are located along the closest walls to the point source, as shown in Fig. 2. This leads to the generation of mirror auxiliary linear sources, which imposes perfect boundary conditions on the two covered walls. In this way, accuracy is preserved for all positions of the source point. Furthermore, efficiency is highly improved, because the values associated to the mirror distribution of sources is known from basic electromagnetic theory [14], [18].

The boundary condition that must fulfill the electric scalar potential along the cavity walls is

$$
\left.G_{V_{\text {rect }}}\left(\vec{r}, \vec{r}_{0,0}^{\prime}\right)\right|_{C}=0
$$




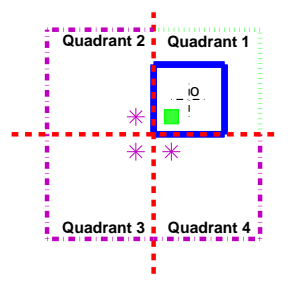

(a) Cavity at first quadrant.

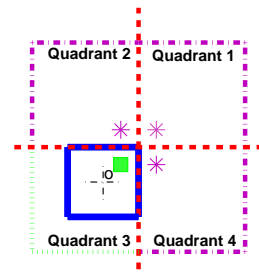

(c) Cavity at third quadrant.

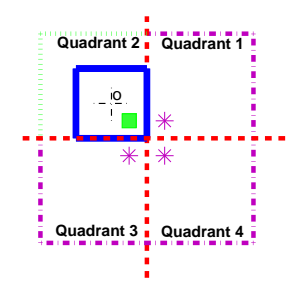

(b) Cavity at second quadrant.

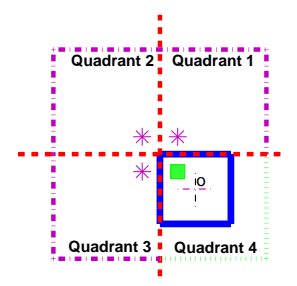

(d) Cavity at fourth quadrant.
Fig. 2: Dynamic position of the auxiliary ground planes as a function of the point source location. The new planes position defines the quadrant where the cavity under analysis is placed. The set of auxiliary linear sources is placed in the same quadrant as the cavity, whereas mirror linear sources appear in all other quadrants.

In order to obtain the values of the auxiliary set of linear charges, $Q\left(\vec{r}^{\prime}\right)$, this last equation is combined with Eq. 1. The resulting equation has the form of an IE [2], which can be solved by the MoM [3]. For this purpose $Q\left(\vec{r}^{\prime}\right)$ is expanded as a sum of basis functions as

$$
Q\left(\vec{r}^{\prime}\right) \approx \sum_{m=1}^{R} \sum_{k=1}^{B_{V}} \alpha_{k, m} \sum_{g=1}^{4} P_{G_{V}}\left(\vec{r}_{0,0}^{\prime}, g\right) f_{V, b, a}^{g, k, m}\left(\vec{r}^{\prime}\right),
$$

where $R$ is the total number of rings, $B_{V}$ is the total number of basis functions, $f_{V, b, a}^{g, k, m}\left(\vec{r}^{\prime}\right)$ is the basis function number $k$, placed on the ring $m$, related to the scalar electric potential $(V)$, and located on any (horizontal or vertical) direction $a$ within the $g$ quadrant (with $g=1,2,3,4$ ), and $\alpha_{k, m}$ is the weight associated to this basis function. Note that a specific weight $\alpha_{k, m}$ is associated to a particular basis function $(k, m)$, but also to all its mirror basis functions (placed in all quadrants, $g=1 \ldots 4)$. The adequate sign of each mirror basis function $\left[P_{G_{V}}\left(\vec{r}_{0,0}^{\prime}, g\right)\right]$ depends on the quadrants of the point source and the mirror images, and it is given in Table I.

Introducing Eq. 6 into the IE, a standard MoM technique yields to a system of linear equations. After solving the system, the weights of the basis functions $\left(\alpha_{k, m}\right)$ are recovered. This allows to express the electric scalar potential inside the multilayered rectangular enclosure as shown in Eq. 7.

In order to compute the $x x$-component of the magnetic vector potential dyadic Green's function, the two boundary conditions to impose are

$$
\begin{aligned}
\left.G_{A_{\text {rect }}}^{x x}\left(\vec{r}, \vec{r}_{0,0}^{\prime}\right)\right|_{C_{h}} & =0, \\
\left.\frac{\partial G_{A_{\text {rect }}}^{x x}\left(\vec{r}, \vec{r}_{0,0}^{\prime}\right)}{\partial x}\right)\left.\right|_{C_{v}} & =0,
\end{aligned}
$$

TABLE I: Signs which must be applied to the auxiliary sources as a function of the quadrants (defined by the ground planes) where the original point source and the auxiliary sources are located.

\begin{tabular}{|c|c|c|c|c|c|}
\multicolumn{2}{c|}{} & \multicolumn{5}{|c|}{ Auxiliary source quadrant [g] } \\
\hline & Source quad. & Quad. 1 & Quad. 2 & Quad. 3 & Quad. 4 \\
\hline \multirow{4}{*}{$P_{G_{V}}$} & 1 & + & - & + & - \\
& 2 & - & + & - & + \\
& 3 & + & - & + & - \\
& 4 & - & + & - & + \\
\hline \multirow{3}{*}{$P_{G}^{x}$} & 1 & + & + & - & - \\
& 2 & + & - & - & + \\
& 3 & - & - & + & + \\
\hline \multirow{3}{*}{$P_{G}^{y}$} & 4 & - & + & + & - \\
& 1 & + & - & - & + \\
& 2 & - & - & + & + \\
& 3 & - & + & + & - \\
& 4 & + & + & - & - \\
\hline
\end{tabular}

where the suffix $h$ and $v$ denote horizontal and vertical walls, respectively. Note that boundary conditions are decoupled and they can be imposed separately on each wall, leading to two systems of equations with $B_{A, \xi}$ unknowns (where $B_{A, \xi}$ is the number of basis function related to the wall $\xi$, with $\xi=h, v$ ) instead of one system of $2 B_{A, \xi}$ unknowns, as formulated in [14]. This is possible because an $x$-oriented dipole can not create a $y$-oriented potential component inside a rectangular cavity, an vice-versa [18]. Introducing the $G_{A}^{x x}$ component (Eq. 4) into Eq. 8 and Eq. 9, two IEs are obtained. In order to apply the MoM, the auxiliary linear distribution of dipoles is expanded as

$$
D\left(\vec{r}^{\prime}\right) \approx \sum_{m=1}^{R} \sum_{\xi=h, v} \sum_{k=1}^{B_{A, \xi}} \alpha_{k, m}^{x, \xi} \sum_{g=1}^{4} P_{G_{A}}^{x}\left(\vec{r}_{0,0}^{\prime}, g\right) f_{A, b, \xi}^{g, k, m}\left(\vec{r}^{\prime}\right)
$$

where $\alpha_{k, m}^{x, \xi}$ is the weight associated to the $k$ basis function, placed at the $m$ ring, associated to the $x$-dipole source and imposed on the $\xi$ wall, and $P_{G_{A}}^{x}\left(\vec{r}_{0,0}^{\prime}, g\right)$ (see Table I) is the sign associated to each auxiliary source, as a function of the quadrant where it is defined.

Introducing Eq. 10 into the two IEs, a standard MoM technique yields to two systems of linear equations. Once they are solved, the weights of the basis functions are recovered, and the $x$-component of the magnetic vector potential can be expressed as shown in Eq. 11. In the case that the source is oriented along the $y$-axis, a similar formulation can easily be derived.

\section{B. Calculation of the $N^{\text {th }}$ order GF derivatives}

The proposed formulation allows the easy computation of the boxed Green's functions spatial derivatives of order $n$, without requiring an additional computational effort. These derivatives may be useful, for instance, during the computation of the fields in the vicinity of microstrip discontinuities or in the analysis of finite size dielectric objects with surface formulations. For this purpose, derivatives are taken on Eq. 7 and Eq. 11 over the source-observer spatial distance $\left(\rho=\left|\vec{r}-\vec{r}^{\prime}\right|\right)$, leading to the expressions shown in Eq. 13 and Eq. 14. The main advantage of this approach is that basis functions and 


$$
\begin{gathered}
G_{V_{\text {rect }}}\left(\vec{r}, \vec{r}_{0,0}^{\prime}\right)=\left.\sum_{g=1}^{4} S_{0}\left[\tilde{G}_{V}\right]\right|_{\vec{r}_{0, g}^{\prime}} ^{\vec{r}_{0}} P_{G_{V}}\left(\vec{r}_{0,0}^{\prime}, g\right)+\left.\sum_{m=1}^{R} \sum_{k=1}^{B_{V}} \alpha_{k, m} \sum_{g=1}^{4} \int_{C_{g}^{\prime}} S_{0}\left[\tilde{G}_{V}\right]\right|_{\vec{r}^{\prime}} ^{\vec{r}} P_{G_{V}}\left(\vec{r}_{0,0}^{\prime}, g\right) f_{V, b, a}^{g, k, m}\left(\vec{r}^{\prime}\right) \partial \vec{r}^{\prime} \\
G_{A_{\text {rect }}}^{x x}\left(\vec{r}, \vec{r}_{0,0}^{\prime}\right)=\left.\sum_{g=1}^{4} S_{0}\left[\tilde{G}_{A}\right]\right|_{\vec{r}_{0, g}^{\prime}} ^{\vec{r}} P_{G_{A}}^{x}\left(\vec{r}_{0,0}^{\prime}, g\right)+\left.\sum_{m=1}^{R} \sum_{\xi=h, v} \sum_{k=1}^{B_{A, \xi}} \alpha_{k, m}^{x, \xi} \sum_{g=1}^{4} \int_{C_{g}^{\prime}} S_{0}\left[\tilde{G}_{A}\right]\right|_{\vec{r}^{\prime}} ^{\vec{r}} P_{G_{A}}^{x}\left(\vec{r}_{0,0}^{\prime}, g\right) f_{A, b, a}^{g, k, m}\left(\vec{r}^{\prime}\right) \partial \vec{r}^{\prime}
\end{gathered}
$$

their associated weights are independent of $(\rho)$, and are not affected by the derivative. This means that there is no need to reformulate the problem for this specific case. On the other hand, the only term in the expressions which is affected by the $(\rho)$ derivative is the Sommerfeld transformation. Specifically, it is known that the derivative of the $N$-order Sommerfeld transformation is related to the $(N+1)$-order Sommerfeld transformation [15], as follows

$$
\frac{\left.\partial S_{N}[\tilde{G}]\right|_{\rho}}{\partial \rho}=\left.S_{N+1}[\tilde{G}]\right|_{\rho}
$$

This property has been employed in Eq. 13 and Eq. 14. Finally, note that the spatial derivatives related to the $x$ or $y$-directions can easily be obtained from these equations, simply by using the chain's rule of the derivative.

\section{Proper Termination of Test and Basis Functions}

The use of auxiliary ground planes requires a proper definition and termination of the basis functions employed in the problem. After that, test functions are located along the noncovered walls of the cavity following similar ideas.

In the case of the electric scalar potential, the auxiliary charge must be zero at the ground planes. This condition is enforced by terminating the mesh with an entire basis function. Besides, the sharp corner on the auxiliary sources (contour $C^{\prime}$ in Fig. 1) is handled by employing two half-basis functions, which are interconnected, creating a unique basis function, to enforce continuity of the charge at the corner. This procedure is similar as the usually employed in the IE MoM for the modeling of junctions in the metalizations [19]. An example of this implementation is shown in Fig. 3.

In the case of the magnetic vector potential, produced by an $x$-oriented source dipole, a zero value must be physically imposed for the auxiliary current at the $x$-oriented ground plane, whereas it does not vanish at the $y$-oriented ground plane [20]. This is modeled by using an additional half-basis function attached to the $y$-oriented plane, and by terminating the mesh with an entire basis function on the $x$-oriented plane. Furthermore, note in Fig. 4 that two independent half-basis functions have been employed to treat the corner. These two half-basis functions are not interconnected, because each of them is referred to a different boundary condition and applied into a different IE. However, the presence of these two half roof-top functions at the corner is important, since the current distribution will in general not be zero at the corner. An example of this implementation is shown in Fig. 4. In the case

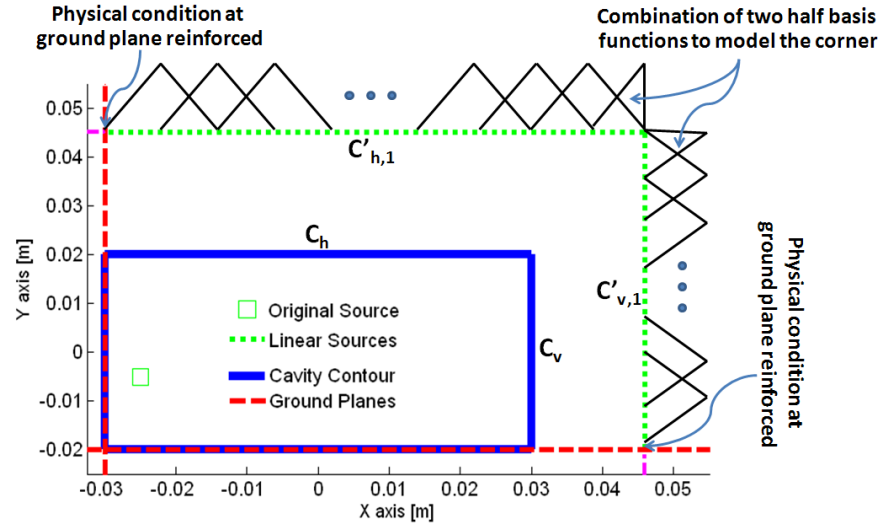

Fig. 3: Example of basis functions (rooftops) definition for the $G_{V}$ computation. The auxiliary linear charge continuity is enforced at the corner using two interconnected half-rooftops (which makes a unique basis function), meanwhile zero values of the charges are enforced at the ground planes.

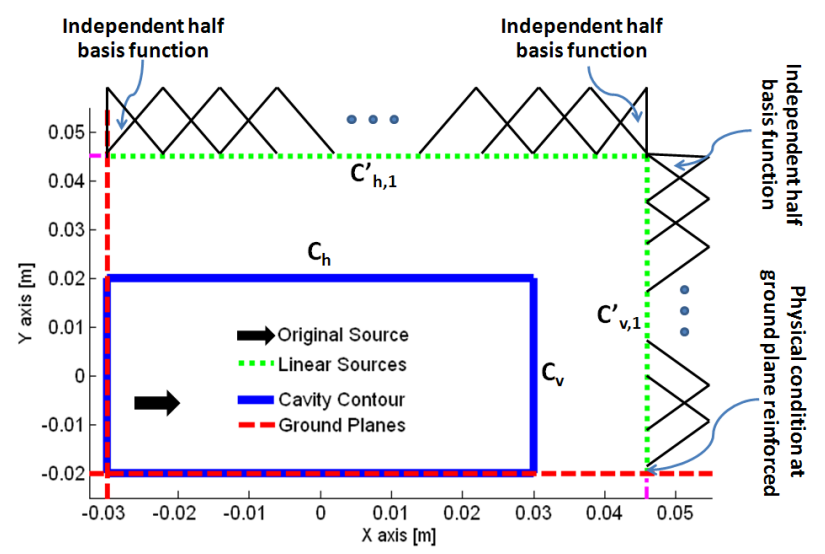

Fig. 4: Example of basis functions (rooftops) definition for the $G_{A}^{x x}$ computation. A zero value of the potential is forced at the $x$-oriented plane by terminating the mesh with a entire basis function. Any value of the potential is allowed at the $y$ oriented plane by inserting there a half-rooftop. The corner is modeled using two isolated half-rooftops (not interconnected).

that the source is a $y$-oriented dipole, an implementation dual to the proposed for the $x$-source is employed.

\section{Basis/Test Functions Choice}

The selection of different basis/test functions provides a trade-off between accuracy and speed in the boxed Green's 


$$
\begin{aligned}
& \frac{\partial^{n} G_{V_{r e c t}}\left(\vec{r}, \vec{r}_{0,0}^{\prime}\right)}{\partial \rho^{n}}=\left.\sum_{g=1}^{4} S_{n-1}\left[\tilde{G}_{V}\right]\right|_{\vec{r}_{0, g}^{\prime}} ^{\vec{r}} P_{G_{V}}\left(\vec{r}_{0,0}^{\prime}, g\right)+\left.\sum_{m=1}^{R} \sum_{k=1}^{B_{V}} \alpha_{k, m} \sum_{g=1}^{4} \int_{C_{g}^{\prime}} S_{n-1}\left[\tilde{G}_{V}\right]\right|_{\vec{r}^{\prime}} ^{\vec{r}} P_{G_{V}}\left(\vec{r}_{0,0}^{\prime}, g\right) f_{V, b, a}^{g, k, m}\left(\vec{r}^{\prime}\right) \partial \vec{r}^{\prime} \\
& \frac{\partial^{n} G_{A_{r e c t}}^{x x}\left(\vec{r}, \vec{r}_{0,0}^{\prime}\right)}{\partial \rho^{n}}=\left.\sum_{g=1}^{4} S_{n-1}\left[\tilde{G}_{A}\right]\right|_{\vec{r}_{0, g}^{\prime}} ^{\vec{r}} P_{G_{A}}^{x}\left(\vec{r}_{0,0}^{\prime}, g\right)+ \\
&\left.\sum_{m=1}^{R} \sum_{\xi=h, v} \sum_{k=1}^{B_{A, \xi}} \alpha_{k, m}^{x, \xi} \sum_{g=1}^{4} \int_{C_{g}^{\prime}} S_{n-1}\left[\tilde{G}_{A}\right]\right|_{\vec{r}^{\prime}} ^{\vec{r}} P_{G_{A}}^{x}\left(\vec{r}_{0,0}^{\prime}, g\right) f_{A, b, a}^{g, k, m}\left(\vec{r}^{\prime}\right) \partial \vec{r}^{\prime}
\end{aligned}
$$

functions computation. Three possible choices are presented and discussed below.

The first option is to use point matching [3]. In this case, the auxiliary linear sources are reduced to spatial images, similar to these introduced in [14], which impose boundary conditions at discrete points on the cavity walls. The main drawback, as in any point-matching technique, is that it leads to ill-conditioned system of equations when the number of unknowns increases. This greatly limits the accuracy of the computed Green's functions. On the other hand, this approach is extremely efficient, because all contour integrals are reduced to a single point evaluation of the function.

The second option uses linear roof-tops as test functions and Dirac-deltas as basis functions (spatial images). This choice presents a trade off between accuracy and speed, but it is not free of instabilities. Accuracy is improved because boundary condition are imposed on the whole cavity perimeter, averaged on each cell. However, ill-conditioned problems arise in the systems of equations when the number of discrete sources increases. In terms of efficiency, the presence of discrete sources reduces the double contour integral to onedimensional integrals, leading to an efficient Green's functions computation.

The third and last option uses linear roof-top test and basis functions. This approach is very accurate and free of illconditioned situations. Specifically, the error committed can arbitrarily be reduced by increasing the number of basis/test functions [3]. The main drawback of this approach is that double contour integrals must be evaluated when imposing the boundary conditions, leading to less efficient computation as compared with the two previous approaches.

\section{NUMERICAL VALIDATION}

This section presents numerical results to validate the proposed technique. For this purpose, the structure shown in Fig. 1 is analyzed. The point source is placed at the position $(-25,-5,3.14) \mathrm{mm}$. The location of the auxiliary linear sources is an important parameter to be considered. In contrast to the approach presented in [13], the impact of this location on the method accuracy is very limited, because the proposed approach is singular-free and the method does not have to compensate for the singular behavior of the original source. Therefore, the method is inherently stable as a function of the location of the auxiliary sources. In spite of this, the

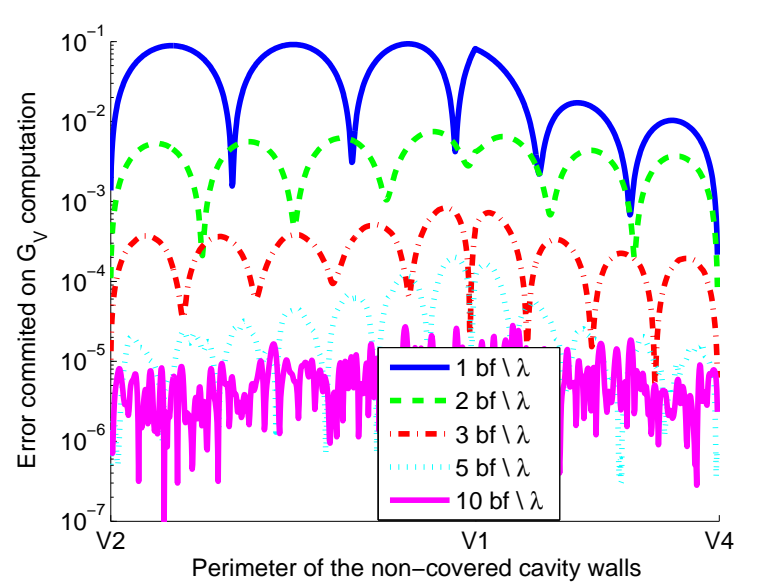

Fig. 5: Error committed in the imposition of the $G_{V}$ boundary conditions (Eq. 5) at $7 \mathrm{GHz}$ along the non-covered walls of the cavity shown in Fig. 1, when different numbers of basis functions (rooftops) per $\lambda$ are employed. $V_{X}$ denotes the $X$ vertex of the cavity, as indicated in Fig. 1.

number of unknowns required to obtain a desired precision varies. If the auxiliary sources are located very close to the cavity walls, their associated singular behavior may degrade the boundary conditions imposition. On the other hand, if the auxiliary sources are located very far away from the walls, the number of unknowns required to achieve a required precision increases, because the auxiliary sources lose effectiveness in representing the fine details of the cavity. Numerical results have shown that values of $d_{x}$ and $d_{y}$ within the range of $0.2 \lambda_{0}-2 \lambda_{0}$ provide good convergence rates using a limited number of test/basis functions. In the following examples, the auxiliary linear sources are always located at the distances $d_{x}=d_{y}=1.5 \lambda$.

The proposed method allows to exactly know the error committed on the Green's functions computation. This can easily be done by evaluating the fulfillment of the boundary conditions along the cavity contour. Since the use of auxiliary ground planes imposes perfect boundary conditions on the covered walls, only the error committed on the two remaining walls must be examined. For the electric scalar potential, this error is obtained by evaluating Eq. 5 along the two noncovered walls. In the case of the magnetic vector potential, 


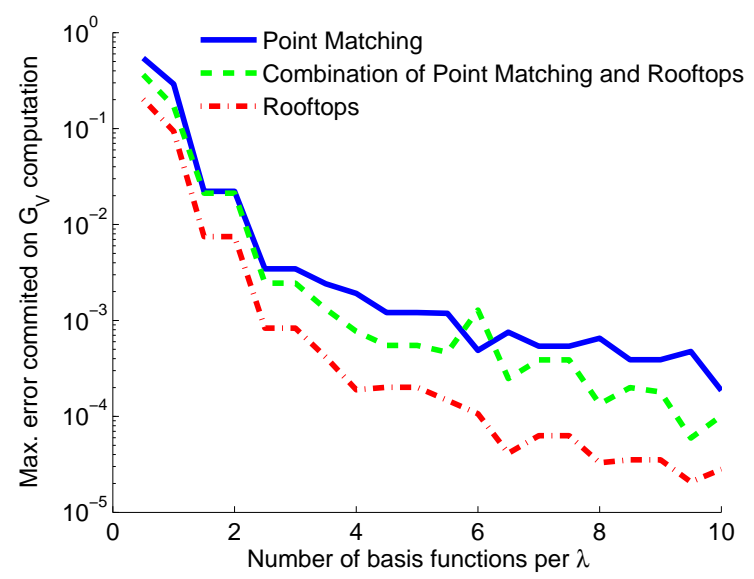

Fig. 6: Maximum error committed in the imposition of the $G_{V}$ boundary conditions at $7 \mathrm{GHz}$ on the cavity shown in Fig. 1 versus the number and type of basis/test functions per $\lambda$ employed.

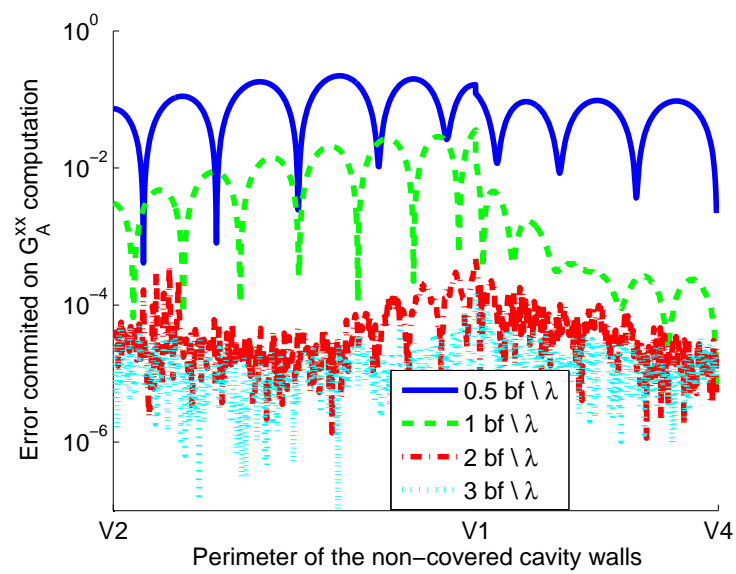

Fig. 7: Error committed on the imposition of the $G_{A}^{x x}$ boundary conditions (Eqs. 8 and 9) at $20 \mathrm{GHz}$ along the non-covered walls of the cavity shown in Fig. 1, when different numbers of basis functions (rooftops) per $\lambda$ are employed. $V_{X}$ denotes the $X$ vertex of the cavity, as indicated in Fig. 1 .

the error is obtained by computing Eq. 8 for the non-covered horizontal wall and Eq. 9 for the non-covered vertical wall. In all cases, an ideal situation will provide a zero value for the relevant condition along the whole cavity perimeter.

First, the error committed in the $G_{V}$ computation at the frequency of $7 \mathrm{GHz}$ is examined. The error is presented for several numbers of basis functions per $\lambda$ (Fig. 5). In this case, linear rooftop test/basis functions have been employed. As can be observed in the figure, the error is small with just one basis function per $\lambda$, and decreases fast as the number of basis functions is increased. This demonstrates that the method is rapidly convergent, requiring a few number of basis functions to obtain very low errors. In Fig. 6 the maximum error (computed over the whole cavity perimeter) is presented for different types and number of basis/test functions. In the

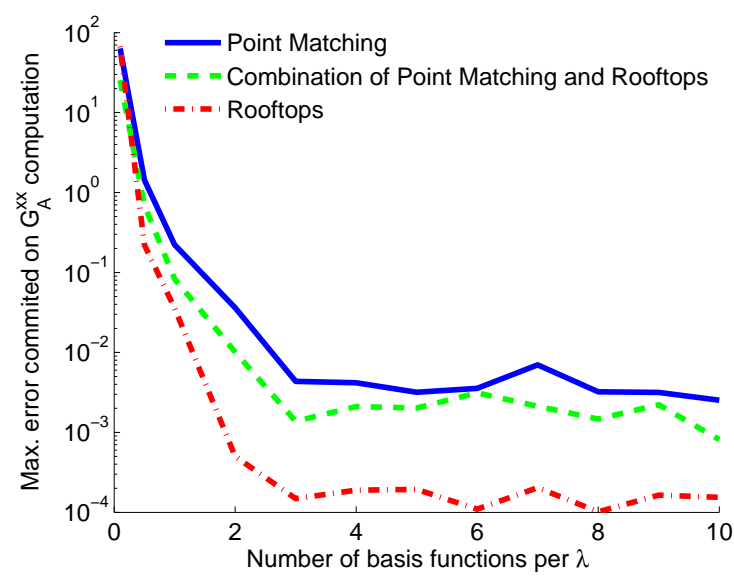

Fig. 8: Maximum error committed in the imposition of the $G_{A}^{x x}$ boundary conditions at $20 \mathrm{GHz}$ on the cavity shown in Fig. 1 versus the number and type of basis/test functions per $\lambda$ employed.

case of point matching, lower convergence rates are found due to its behavior inside IEs [3]. The use of combined roof-tops and delta Dirac test/basis functions improves the convergence rates. Note that these two approaches lead to ill-conditioned situations when the number of basis functions increases more. Finally, the use of linear roof-tops as basis/test functions presents the best convergence rates, thanks to the use of continuous auxiliary sources. We have verified that the use of 500 basis/test functions provides an error within the precision of the computer along the complete perimeter, showing that the method is inherently stable.

Second, the error committed on the $G_{A}^{x x}$ computation is considered. In this case, the frequency is set to $20 \mathrm{GHz}$ in order to fully appreciate the influence of the lateral walls on this potential. Fig. 7 shows the error along the cavity contour when different numbers of basis functions (rooftops) are employed. Finally, in Fig. 8 a comparison of the maximum error committed, depending on the number and type of test/basis functions, is presented. The analysis of these results leads to the same conclusions as given for the $G_{V}$ case, and confirms the effectiveness of the proposed approach.

Note that the accuracy of the technique also depends on the accuracy of the method employed to compute the Sommerfeld transformation [15]. Therefore, it is important to avoid errors in these transformations, which may accumulate and propagate towards the Green's functions. This occurs in Fig. 8, where the error of $10^{-4}$ on the $G_{A}^{x x}$ computation is fixed by the maximum error while calculating $S_{1}$.

\section{Analysis of Multilayered Shielded Circuits}

In this section, the proposed spatial technique is applied to the fast analysis of practical multilayered shielded circuits. For this purpose, the method has efficiently been included into a MPIE formulation [2], following the acceleration technique introduced in [13]. Then, two shielded microstrip filters are taken from the literature and are analyzed by the proposed technique. The obtained results are compared, in terms of 


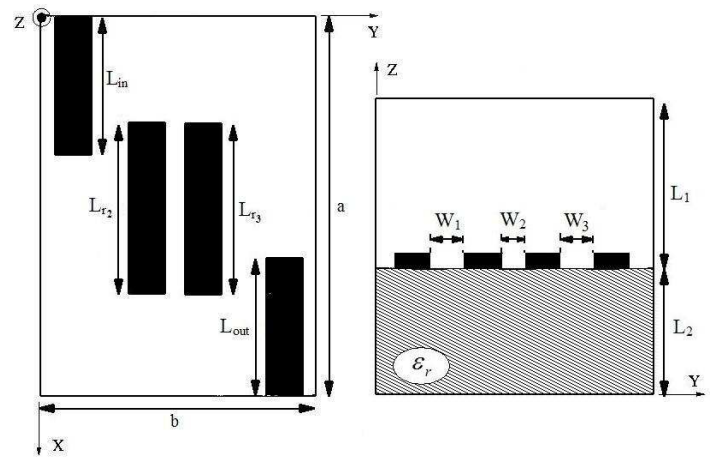

Fig. 9: Multi-band filter in hybrid waveguide-microstrip technology, reproduced from [21]. $L_{1}=3.0 \mathrm{~mm}, L_{2}=3.15 \mathrm{~mm}$, $\epsilon_{r}=2.2 \mathrm{~mm}, L_{\text {in }}=L_{\text {out }}=14.0 \mathrm{~mm}, L_{r 1}=L_{r 2}=24.54$ $\mathrm{mm}, W_{1}=W_{3}=1.8 \mathrm{~mm}, W_{2}=5.0 \mathrm{~mm}, a=40.0 \mathrm{~mm}$, $b=34.0 \mathrm{~mm}$.

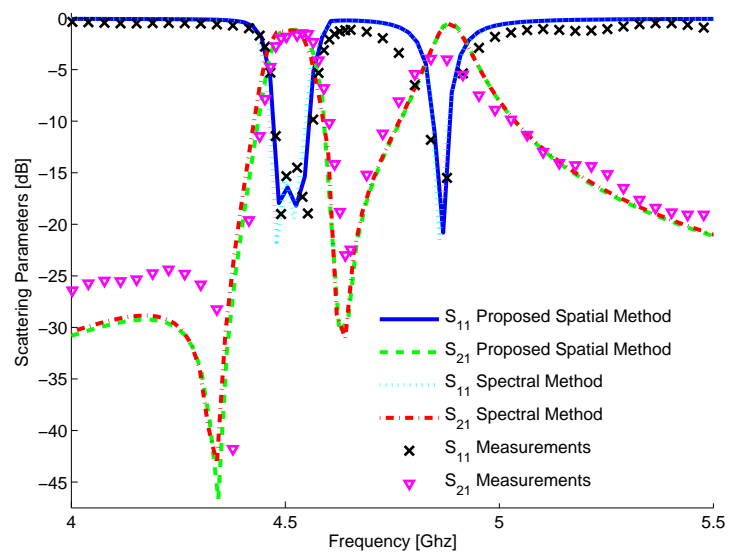

Fig. 10: Results for the bandpass filter shown in Fig. 9, analyzed using the proposed spatial approach and validated by the spectral method presented in [11].

TABLE II:

Comparison of the time (per frequency point) required by the proposed spatial method and a spectral technique [11] for the analysis of the filter shown in Fig. 9.

\begin{tabular}{|c|cc|cc|}
\multicolumn{1}{|c|}{} & \multicolumn{2}{|c|}{ Proposed Spatial Method } & \multicolumn{2}{c|}{ Spectral Method [11] } \\
\hline Mesh & Time per $f . p$. & Bf per $\lambda$ & Time per $f . p$. & Modes \\
\hline 45 cells & $0.108 \mathrm{sec}$ & 3.0 & $0.075 \mathrm{sec}$ & 2500 \\
\hline 90 cells & $0.312 \mathrm{sec}$ & 3.0 & $0.665 \mathrm{sec}$ & 2500 \\
\hline 135 cells & $0.604 \mathrm{sec}$ & 3.0 & $1.903 \mathrm{sec}$ & 3500 \\
\hline 180 cells & $1.012 \mathrm{sec}$ & 3.0 & $3.909 \mathrm{sec}$ & 3500 \\
\hline
\end{tabular}

accuracy and efficiency, against a spectral domain approach [11] and against measurements. Note that the comparison has been carried out exactly in the same conditions, including computer (Pentium IV, $2.5 \mathrm{GHz}, 2 \mathrm{~Gb}$ RAM), mesh, and integration points.

In the literature, the analysis of shielded microwave filters have mainly been performed in the spectral domain [9], [10], [11]. This formulation is usually very efficient, but has some important convergence problems when the dimension of the

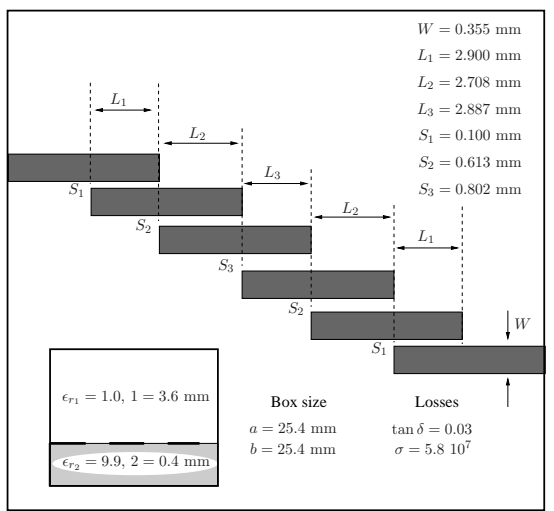

Fig. 11: Boxed microstrip bandpass filter of fourth order, based on coupled line sections, reproduced from [11].

cells employed to discretize the printed circuits are very small as compared with the enclosure. In this case, the number of modes required to achieve convergence is very high, and there is not a clear rule to truncate the infinite summations. On the other hand, the use of the spatial domain formulation for this type of analysis is not practical, due to the high computational cost that requires the calculation of multilayered boxed Green's functions [5] [6] [7].

The first filter under consideration is shown in Fig. 9 [21]. The results of the analysis employing the proposed spatial technique, and a spectral approach [11] are presented in Fig. 10. Measured data is included as validation. Note that an extraordinary agreement between the two completely different methods has been obtained. This structure is specially difficult to be handled by the spatial method, because it has a thick substrate and it requires two rings of auxiliary linear sources. A careful study about the efficiency of the two methods, as a function of the number of discretization cells, is presented in Table II. As can be observed, the proposed spatial technique completely converges using 3 basis functions per $\lambda$, independently of the mesh. As expected, the spectral method requires a higher number of modes to converge as the mesh density increases. In terms of efficiency, the proposed spatial method is able to compete against the spectral approach in all cases. For low mesh density, the spectral approach is slightly faster, because it converges summing up a low number of modes. However, as the mesh density increases, the spatial approach becomes more and more efficient (even two and three times faster). This is because an increase in the mesh density only affects the size of the MoM matrix, but it does not affect to the speed in the calculation of the Green's functions.

The second filter considered is shown in Fig. 11 [11]. The results of the analysis are presented in Fig. 12, where again an extraordinary agreement has been found between the proposed spatial technique and the spectral approach [11]. This structure is specially difficult to be handled by the spectral method, because it has a large box as compared with the size of the printed circuits. Table III presents a careful study related to the efficiency and convergence of both techniques. Again, the proposed spatial method converges in all cases using just 3 


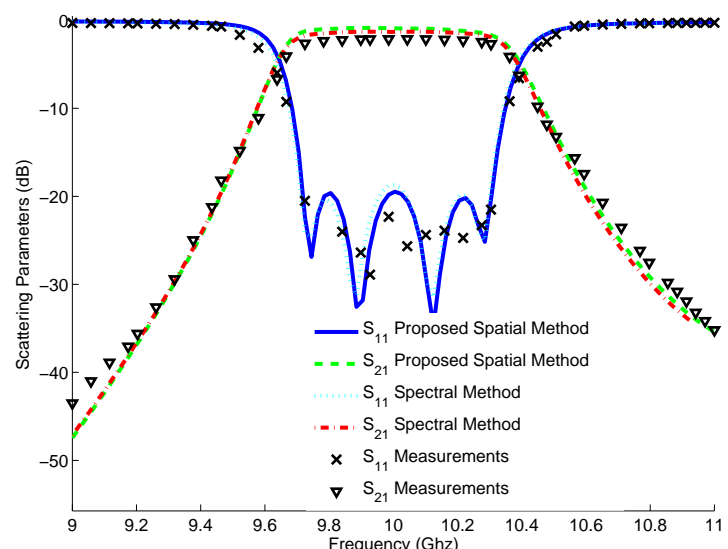

Fig. 12: Results for the bandpass filter shown in Fig. 11, analyzed using the proposed spatial approach and validated by the spectral method presented in [11].

\section{TABLE III:}

Comparison of the time (per frequency point) required by the proposed spatial method and a spectral technique [11] for the analysis of the filter shown in Fig. 11.

\begin{tabular}{|c|cc|cc|}
\multicolumn{1}{c|}{} & \multicolumn{2}{c|}{ Proposed Spatial Method } & \multicolumn{2}{c|}{ Spectral Method [11] } \\
\hline Mesh & Time per $f . p$. & Bf per $\lambda$ & Time per $f \cdot p \cdot$ & Modes \\
\hline 38 cells & $0.072 \mathrm{sec}$ & 3.0 & $0.160 \mathrm{sec}$ & 20000 \\
\hline 76 cells & $0.184 \mathrm{sec}$ & 3.0 & $1.209 \mathrm{sec}$ & 22500 \\
\hline 114 cells & $0.328 \mathrm{sec}$ & 3.0 & $9.570 \mathrm{sec}$ & 40000 \\
\hline 152 cells & $0.512 \mathrm{sec}$ & 3.0 & $18.591 \mathrm{sec}$ & 50000 \\
\hline
\end{tabular}

basis functions per $\lambda$. On the contrary, the convergence of the spectral method directly depends on the size of the mesh, requiring a very large number of modes in all cases. In terms of efficiency, the proposed spatial technique is always much faster than the spectral approach. For low mesh densities, even though both techniques are quite competitive, the spatial method is more than two times faster. The efficiency distance between the two methods increases with the mesh density, being the spatial technique more than 35 times faster than the spectral approach for the case of a very dense mesh.

Finally, it is important to remark that the proposed spatial technique provides a stable and systematic approach to efficiently analyze any shielded microwave device. As a difference with the spectral domain approach, the proposed method is able to obtain convergent results for any cavity and printed circuit sizes. Consequently, the proposed technique is an excellent candidate to be integrated into a CAD tool, for the fast analysis and efficient optimization of practical shielded multilayered printed circuits.

\section{CONCLUSIONS}

A grounded MoM-based spatial domain technique has been presented for the Green's functions analysis of multilayered rectangular enclosures. The technique combines the use of auxiliary ground planes, which cover two walls of the cavity, with a set of auxiliary linear distribution of sources employed to impose the boundary conditions along the cavity contour.
Mirror linear sources appear with respect to the planes, perfectly imposing boundary conditions on the two covered walls. On the other two walls, a numerical imposition of these conditions has led to a set of integral equations. A convergence study, related to the test and basis functions choice, has been presented and discussed. Finally, the computed Green's functions have been included into an MPIE formulation, for the accurate and very fast analysis of practical multilayered shielded circuits. A rigorous comparative between different IE methods has demonstrated that the proposed method is ideal for the analysis, design and optimization of shielded circuits, in terms of convergence, accuracy and efficiency.

\section{ACKNOWLEDGEMENT}

This work was partially supported by the Spanish Ministry of Education and Science under Grant FPU-AP2006-015 and with the Project TEC2007-67630-C03-02.

\section{REFERENCES}

[1] L. P. Dunleavy and P. B. Katehi, "Shielding effects in microstrip discontinuities," IEEE Transactions on Microwave Theory and Techniques, vol. 36, no. 12, pp. 1767-1774, December 1988.

[2] J. R. Mosig, Integral Equation Technique. New York: Wiley Interscience Publication, 1989.

[3] R. F. Harrington, Field Computation by Moment Methods. New York: The MacMillan Company, 1968.

[4] R. H. Jansen, "The spectral-domain approach for microwave integrated circuits," IEEE Transactions on Microwave Theory and Techniques, vol. 33, p. 10431056, October 1985.

[5] L. P. Dunleavy and P. B. Katehi, "A generalized method for analyzing shielded thin microstrip discontinuities," IEEE Transactions on Microwave Theory and Techniques, vol. 36, no. 12, pp. 1758-1766, December 1988.

[6] M.-J. Park and S. Nam, "Rapid calculation of the Green's function in the shielded planar structures," IEEE Microwave and Guided Wave Letters, vol. 7, no. 10, pp. 326-328, October 1997.

[7] A. Álvarez Melcón and J. R. Mosig, "Two techniques for the efficient numerical calculation of the Green's functions for planar shielded circuits and antennas," IEEE Transactions on Microwave Theory and Techniques, vol. 48, no. 9, pp. 1492-1504, September 2000.

[8] J. L. Tsalamengas and G. Fikioris, "Rapidly converging spectral-domain analysis of rectangularly shielded layered microstrip lines," IEEE Transactions on Microwave Theory and Techniques, vol. 51, no. 6, pp. 17291734, June 2005.

[9] C. J. Railton and S. A. Meade, "Fast rigorous analysis of shielded planar filters," IEEE Transactions on Microwave Theory and Techniques, vol. 40, no. 5, pp. 978-985, May 1992.

[10] G. V. Eleftheriades, J. R. Mosig, and M. Guglielmi, "A fast integral equation technique for shielded planar circuits defined on nonuniform meshes," IEEE Transactions on Microwave Theory and Techniques, vol. 44, no. 12, pp. 2293-2296, December 1996.

[11] A. Álvarez Melcón, J. R. Mosig, and M. Guglielmi, "Efficient CAD of boxed microwave circuits based on arbitrary rectangular elements," IEEE Transactions on Microwave Theory and Techniques, vol. 47, no. 7, pp. 1045-1058, July 1999.

[12] F. D. Quesada Pereira, P. Vera Castejón, D. Cañete Rebenaque, J. Pascual García, and A. Álvarez Melcón, "Numerical evaluation of the Green's functions for cylindrical enclosures," IEEE Transactions on Microwave Theory and Techniques, vol. 53, no. 1, pp. 94-105, January 2005.

[13] J. S. Gómez-Díaz, M. Martínez-Mendoza, F. J. Pérez-Soler, F. D. Quesada-Pereira, and A. Álvarez-Melcón, "Practical Implementation of the Spatial Images Technique for the Analysis of Shileded Multilayered Printed Circuits," IEEE Transactions on Microwave Theory and Techniques, vol. 56, no. 1, pp. 131-141, January 2008.

[14] J. S. Gómez-Díaz, M. García-Vigueras, D. Cañete-Rebenaque, F. D. Quesada-Pereira, and A. Álvarez-Melcón, "Use of Ground Planes within the Spatial Images Technique: Application to the Analysis of Rectangular Multilayered Shielded Enclosures," in IEEE International Microwave Symposium, Anaheim, California, USA, 23-28 May 2010. 
[15] K. Michalski, "Extrapolation methods for Sommerfeld integral tails," IEEE Transactions on Antennas and Propagation, vol. 46, no. 10, pp. 1405-1418, October 1998.

[16] T. Itoh, Numerical Techniques for Microwave and Millimeter-Wave Passive Structures. Wiley-Interscience, 1989.

[17] F. D. Quesada Pereira, P. Vera Castejón, J. L. Gómez Tornero, D. Cañete Rebenaque, J. Pascual García, and A. Álvarez Melcón, "Analysis of microstrip to circular waveguide transitions by a new spatial images method," Microwave and Optical Technology Letters, vol. 45, pp. 563568, June 2005.

[18] C. A. Balanis, Advanced Engineering Electromagnetics. John Wiley and Sons, 1989.

[19] B. Kolundzija and A. Djordjevic, Eds., Electromagnetic Modeling of Composite Metallic and Dielectric Structures. Artech House Publishers, 2002 .

[20] C. A. Balanis, Antenna Theory: Analysis and Design. 3rd Edition. John Wiley and Sons, 2005.

[21] M. Martínez-Mendoza, J. S. Gómez-Díaz, D. Cañete-Rebenaque, and A. Álvarez-Melcón, "Design of Dual-Bandpass Hybrid WaveguideMicrostrip Microwave Filters," IEEE Transactions on Microwave Theory and Techniques, vol. 56, no. 12, pp. 2913-2920, December 2008. 